Vol. 1, Issue 1, pp. 022-027

\title{
CONSTRUCTION OF A SUMMATION FORMULA INTERLACED WITH CONTIGUOUS RELATION
}

\section{SALAHUDDIN}

P.D.M College of Engineering, Bahadurgarh, Haryana, India

\section{Abstract}

The main aim of this paper is to develop a summation formula interlaced with Hypergeometric function and contiguous relation. 2010 MSC NO : 33C05 , 33C20 , 33D15, 33D50 , 33D60

Key words: Contiguous relation, Recurrence relation, Legendre's duplication formula.

\section{A Introduction}

Generalized Gaussian Hypergeometric function of one variable

$$
{ }_{\mathrm{A}} \mathrm{F}_{\mathrm{B}}\left(\mathrm{a}_{1}, \mathrm{a}_{2}, \ldots, \mathrm{a}_{\mathrm{A}} ; \mathrm{b}_{1}, \mathrm{~b}_{2}, \ldots \mathrm{b}_{\mathrm{B}} ; \mathrm{z}\right)=\sum_{k=0}^{\infty} \frac{\left(\mathrm{a}_{1}\right)_{k}\left(\mathrm{a}_{2}\right)_{k} \ldots .\left(\mathrm{a}_{\mathrm{A}}\right)_{k} \mathrm{z}^{\mathrm{k}}}{\left(b_{1}\right)_{k}\left(b_{2}\right)_{k} \ldots . .\left(b_{B}\right)_{k} k !}
$$

where the parameters $b_{1}, b_{2}, \ldots, b_{B}$ are neither zero nor negative integers and $A, B$ are non negative integers.

\section{Contiguous Relation :}

[Abramowitz p.558(15.2.19)]

(a-b) $(1-\mathrm{z}){ }_{2} \mathrm{~F}_{1}(\mathrm{a}, \mathrm{b} ; \mathrm{c} ; \mathrm{z})=(\mathrm{c}-\mathrm{b}){ }_{2} \mathrm{~F}_{1}(\mathrm{a}, \mathrm{b}-1 ; \mathrm{c} ; \mathrm{z})+(\mathrm{a}-\mathrm{c}){ }_{2} \mathrm{~F}_{1}(\mathrm{a}-1, \mathrm{~b} ; \mathrm{c} ; \mathrm{z})$

Legendre's duplication formula :

$$
\begin{aligned}
& \Gamma(2 z)=2^{2 z-1} \frac{\Gamma(z) \Gamma\left(z+\frac{1}{2}\right)}{\sqrt{\pi}}, z \neq 0,-1,-2 \\
& \Gamma\left(\frac{1}{2}\right)=\sqrt{\pi}=2^{b-1} \frac{\Gamma\left(\frac{b+1}{2}\right) \Gamma\left(\frac{b}{2}\right)}{\Gamma(b)}
\end{aligned}
$$

\section{A New Summation Formula}

$$
\begin{aligned}
& \text { [A sish et.al. } p \cdot 337(10)] \\
& { }_{2} F_{1}\left(a, b ; \frac{a+b-1}{2} ; \frac{1}{2}\right)=2^{b-1} \frac{\Gamma\left(\frac{a+b-1}{2}\right)}{\Gamma(b)} \quad\left[\frac{\Gamma\left(\frac{b}{2}\right)}{\Gamma\left(\frac{2-1}{2}\right)}\left\{\frac{b+a-1}{a-1}\right\}+2 \frac{\Gamma\left(\frac{b+1}{2}\right)}{\Gamma\left(\frac{2}{2}\right)}\right]
\end{aligned}
$$

\section{$\underline{\text { Recurrence relation }}$}

$$
\Gamma(\zeta+1)=\zeta \Gamma(\zeta)
$$

\section{B MAIN SUMMATION FORMULAE}

For the formula $\mathrm{a} \neq \mathrm{b}$

$$
{ }_{2} \mathrm{~F}_{1}\left(\mathrm{a}, \mathrm{b} ; \frac{\mathrm{a}+\mathbf{b}-\mathbf{3 7}}{2} ; \frac{\mathbf{1}}{\mathbf{2}}\right)=2^{\mathrm{b}-1} \frac{\Gamma\left(\frac{\mathrm{a}+\mathbf{b}-37}{\mathbf{2}}\right)}{(\mathrm{a}-\mathrm{b}) \Gamma(\mathrm{b})}\left[\frac { \Gamma ( \frac { \mathbf { b } } { \mathbf { 2 } } ) } { \Gamma ( \frac { \mathrm { a } - 3 7 } { 2 } ) } \left\{\frac{-8200794532637891559375 \mathrm{a}}{\prod_{\Psi=1}^{19}\{\mathrm{a}-(2 \Psi-1)\}}\right.\right.
$$




\section{SALAHUDDIN / IOSR Journal of Engineering (IOSRJEN)}

www.iosrjen.org

Vol. 1, Issue 1, pp. 022-027

$20125013723397976152375 \mathrm{a}^{2}-19688993487602867898225 \mathrm{a}^{3}+10792700030471840300745 \mathrm{a}^{4}$ $\prod_{\Psi=1}^{19}\{\mathrm{a}-(2 \Psi-1)\}$

$-3824294822931302783964 a^{5}+946995223404049011324 a^{6}-171930790626988570804 a^{7}$

$$
\Pi_{\Psi=1}^{19}\{a-(2 \Psi-1)\}
$$

$23615262213846406804 a^{8}-2505874787291646498 a^{9}+208251057899323218 a^{10}-13663776163658478 a^{11}$ $\prod_{\Psi=1}^{19}\{a-(2 \Psi-1)\}$

$710084079834558 a^{12}-29186718196012 a^{13}+942715036492 a^{14}-23625216132 a^{15}+449681892 a^{16}$ $\prod_{\Psi=1}^{19}\{\mathrm{a}-(2 \Psi-1)\}$

$-6278151 a^{17}+60591 a^{18}-361 a^{19}+a^{20}+8200794532637891559375 b-29248961707347868727325 a^{2} b$ $\prod_{\Psi=1}^{19}\{a-(2 \Psi-1)\}$

$37851190175224311447870 a^{3} \mathrm{~b}-21940789525204753208628 \mathrm{a}^{4} \mathrm{~b}+8502383149162038952848 \mathrm{a}^{5} \mathrm{~b}$ $\prod^{19}\{1 \mathrm{a}-(2 \Psi-1)\}$

$+2040060627807052600148 a^{6} b+399455532659455476552 a^{7} b-51162010304921997390 a^{8} b$ $\prod_{1=1}^{19}\{a-(2 \Psi-1)\}$

$5906444348989582320 \mathrm{a}^{9} \mathrm{~b}-442904964889302630 \mathrm{a}^{10} \mathrm{~b}+32180938804379508 \mathrm{a}^{11} \mathrm{~b}-1452739207922756 \mathrm{a}^{12} \mathrm{~b}$ $\prod_{\Psi=1}^{19}\{a-(2 \Psi-1)\}$

$67865112823536 \mathrm{a}^{13} \mathrm{~b}-1806276624516 \mathrm{a}^{14} \mathrm{~b}+53481117768 \mathrm{a}^{15} \mathrm{~b}-767573001 \mathrm{a}^{16} \mathrm{~b}+13527696 \mathrm{a}^{17} \mathrm{~b}$ $\prod_{\Psi=1}^{19}\{\mathrm{a}-(2 \Psi-1)\}$

$-79781 \mathrm{a}^{18} \mathrm{~b}+702 \mathrm{a}^{19} \mathrm{~b}-20125013723397976152375 \mathrm{~b}^{2}+29248961707347868727325 \mathrm{ab}$ $\prod_{\Psi=1}^{19}\{a-(2 \Psi-1)\}$

$-19800075528365005357848 a^{3} b^{2}+16622611834183848417132 a^{4} b^{2}-6266048334316896142212 a^{5} b^{2}$ $\prod_{\Psi=1}^{19}\{\mathrm{a}-(2 \Psi-1)\}$

$1836520912680391333656 a^{6} b^{2}-307201532349114442312 a^{7} b^{2}+48921372698387829926 a^{8} b^{2}$ $\prod_{\Psi=1}^{19}\{a-(2 \Psi-1)\}$

$-4437363066377882498 a^{9} b^{2}+434677894485480360 a^{10} b^{2}-22693604020866792 a^{11} b^{2}$ $\prod_{\Psi_{1}^{19}}^{19}\{a-(2 \Psi-1)\}$

$1437536850798412 \mathrm{a}^{12} \mathrm{~b}^{2}-42900139812068 \mathrm{a}^{13} \mathrm{~b}^{2}+1777083009832 \mathrm{a}^{14} \mathrm{~b}^{2}-27936753208 \mathrm{a}^{15} \mathrm{~b}^{2}$ $\prod_{\Psi^{1}=1}^{9}\{a-(2 \Psi-1)\}$

$738751065 a^{16} b^{2}-4688307 a^{17} b^{2}+73112 a^{18} b^{2}+19688993487602867898225 b^{3}$ $\prod^{19}+\{a-(2 \Psi-1)\}$

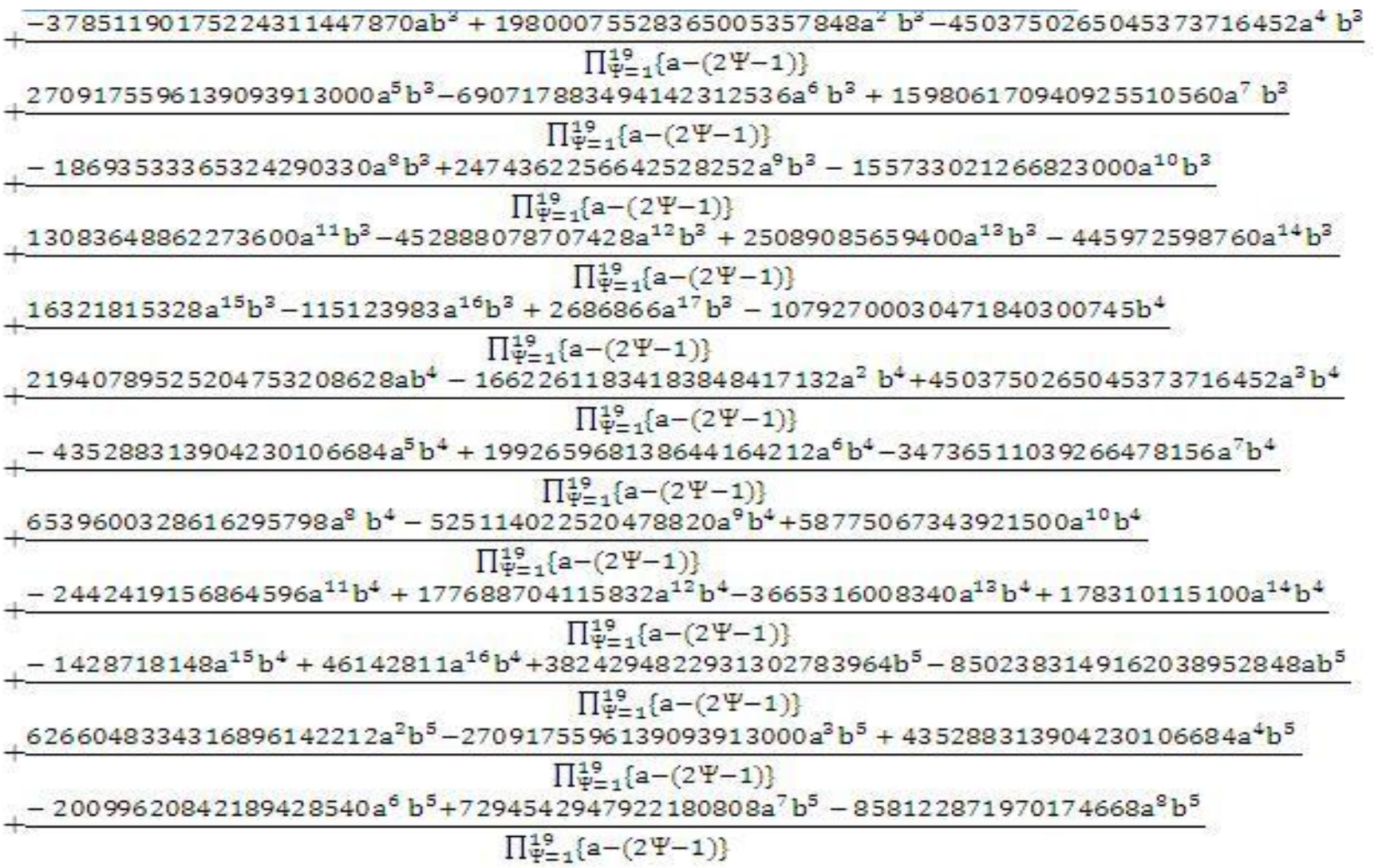


SALAHUDDIN / IOSR Journal of Engineering (IOSRJEN)

www.iosrjen.org

Vol. 1, Issue 1, pp. 022-027

$+134499059719895728 a^{9} b^{5}-7068114134989908 a^{10} b^{5}+678335525811944 a^{11} b^{5}-16734032518476 a^{12} b^{5}$

$\prod_{\Psi_{1}}^{19}\{a-(2 \Psi-1)\}$

$1062990012896 a^{13} b^{5}-9882951156 a^{14} b^{5}+423830264 a^{15} b^{5}-946995223404049011324 b^{6}$

$\prod_{\Psi_{1}^{19}}^{19}\{a-(2 \Psi-1)\}$

$+2040060627807052600148 a b^{6}-1836520912680391333656 a^{2} b^{6}+690717883494142312536 a^{3} b^{6}$

$\prod_{\Psi_{1}}^{19}\{a-(2 \Psi-1)\}$

$-199265968138644164212 a^{4} b^{6}+20099620842189428540 a^{5} b^{6}-467892286162441712 a^{7} b^{6}$

$\prod_{\Psi^{19}}^{19}\{\mathrm{a}-(2 \Psi-1)\}$

$+138686382323023964 a^{8} b^{6}-10538184531582708 a^{9} b^{6}+1399987726233096 a^{10} b^{6}-43367983002344 a^{11} b^{6}$ $\prod_{\Psi=1}^{19}\{a-(2 \Psi-1)\}$

$3609666033428 a^{12} b^{6}-40010466076 a^{13} b^{6}+2234741392 a^{14} b^{6}+171930790626988570804 b^{7}$ $\prod_{\Psi_{1}}^{19}\{\mathrm{a}-(2 \Psi-1)\}$

$+-399455532659455476552 a^{7}+307201532349114442312 a^{2} b^{7}-159806170940925510560 a^{3} b^{7}$

$\prod_{\Psi}^{19}\{a-(2 \Psi-1)\}$

$34736511039266478156 a^{4} b^{7}-7294542947922180808 a^{5} b^{7}+467892286162441712 a^{6} b^{7}$

$\prod_{\Psi^{\prime}=1}^{19}\{a-(2 \Psi-1)\}$

$-5502741133253364 a^{8} b^{7}+1362724070798376 a^{9} b^{7}-60417876724920 a^{10} b^{7}+6902195972256 a^{11} b^{7}$ $\prod_{\Psi=1}^{19}\{a-(2 \Psi-1)\}$

$-95535194508 a^{12} b^{7}+6962078952 a^{13} b^{7}-23615262213846406804 b^{8}+51162010304921997390 a b^{8}$ $\prod_{\Psi^{\prime}=1}^{19}\{a-(2 \Psi-1)\}$

$+-48921372698387829926 \mathrm{a}^{2} \mathrm{~b}^{8}+18693533365324290330 \mathrm{a}^{3} \mathrm{~b}^{8}-6539600328616295798 \mathrm{a}^{4} \mathrm{~b}^{8}$

$\prod_{\Psi=1}^{19}\{a-(2 \Psi-1)\}$

$858122871970174668 a^{5} b^{8}-138686382323023964 a^{6} b^{8}+5502741133253364 a^{7} b^{8}-30542424219450 a^{9} b^{8}$ $\prod_{\Psi=1}^{19}\{a-(2 \Psi-1)\}$

$6433836899490 a^{10} b^{8}-126443639790 a^{11} b^{8}+12570420330 a^{12} b^{8}+2505874787291646498 b^{9}$ $\prod_{\Psi=1}^{19}\{a-(2 \Psi-1)\}$

$-5906444348989582320 a b^{9}+4437363066377882498 a^{2} b^{9}-2474362256642528252 a^{3} b^{9}$

$\prod_{\Psi^{1}=1}^{19}\{a-(2 \Psi-1)\}$

$525114022520478820 a^{4} b^{9}-134499059719895728 a^{5} b^{9}+10538184531582708 a^{6} b^{9}-1362724070798376 a^{7} b^{9}$ $\prod_{\Psi=1}^{19}\{a-(2 \Psi-1)\}$

$30542424219450 a^{8} b^{9}-62359143990 a^{10} b^{9}+11338026180 a^{11} b^{9}-208251057899323218 b^{10}$

$$
\prod_{\Psi^{\prime}=1}^{19}\{a-(2 \Psi-1)\}
$$

$442904964889302630 \mathrm{ab}^{10}-434677894485480360 \mathrm{a}^{2} \mathrm{~b}^{10}+155733021266823000 \mathrm{a}^{3} \mathrm{~b}^{10}$

$\prod_{\Psi=1}^{19}\{a-(2 \Psi-1)\}$

$-58775067343921500 a^{4} b^{10}+7068114134989908 a^{5} b^{10}-1399987726233096 a^{6} b^{10}+60417876724920 a^{7} b^{10}$ $\prod_{\Psi=1}^{19}\{\mathrm{a}-(2 \Psi-1)\}$

$-6433836899490 a^{8} b^{10}+62359143990 a^{9} b^{10}+13663776163658478 b^{11}-32180938804379508 a b^{11}$

$\prod_{\Psi=1}^{19}\{a-(2 \Psi-1)\}$

$+22693604020866792 a^{2} b^{11}-13083648862273600 a^{3} b^{11}+2442419156864596 a^{4} b^{11}-678335525811944 a^{5} b^{11}$

$\prod_{\Psi=1}^{19}\{\mathrm{a}-(2 \Psi-1)\}$

$43367983002344 a^{6} b^{11}-6902195972256 a^{7} b^{11}+126443639790 a^{8} b^{11}-11338026180 a^{9} b^{12}$

$\prod_{\Psi^{19}=1}^{19}\{\mathrm{a}-(2 \Psi-1)\}$

$-710084079834558 b^{12}+1452739207922756 a b^{12}-1437536850798412 a^{2} b^{12}+452888078707428 a^{3} b^{12}$

$\prod_{\Psi^{\prime}=1}^{19}\{a-(2 \Psi-1)\}$

$+-177688704115832 a^{4} b^{12}+16734032518476 a^{5} b^{12}-3609666033428 a^{6} b^{12}+95535194508 a^{7} b^{12}$

$\prod_{\Psi=1}^{19}\{a-(2 \Psi-1)\}$

$+-12570420330 a^{8} b^{12}+29186718196012 b^{13}-67865112823536 a b 613+42900139812068 a^{2} b^{13}$

$\prod_{\Psi=1}^{19}\{a-(2 \Psi-1)\}$

$+-25089085659400 a^{3} b^{13}+3665316008340 a^{4} b^{13}-1062990012896 a^{5} b^{13}+40010466076 a^{6} b^{13}$

$\prod_{\Psi=1}^{19}\{a-(2 \Psi-1)\}$

$+-6962078952 a^{7} b^{13}-942715036492 b^{14}+1806276624516 a b^{14}-1777083009832 a^{2} b^{14}+445972598760 a^{3} b^{14}$ $\prod_{\Psi=1}^{19}\{a-(2 \Psi-1)\}$

$+-178310115100 a^{4} b^{14}+9882951156 a^{5} b^{14}-2234741392 a^{6} b^{14}+23625216132 b^{15}-53481117768 a b^{15}$

$\prod_{\Psi^{\prime}=1}^{19}\{a-(2 \Psi-1)\}$ 
Vol. 1, Issue 1, pp. 022-027

$27936753208 a^{2} b^{15}-16321815328 a^{3} b^{15}+1428718148 a^{4} b^{15}-423830264 a^{5} b^{15}-449681892 b^{16}$ $\prod_{\Psi=1}^{19}\{a-(2 \Psi-1)\}$

$767573001 a b^{16}-738751065 a^{2} b^{16}+115123983 a^{3} b^{16}-46142811 a^{4} b^{16}+6278151 b^{17}-13527696 a b^{17}$ $\prod_{\Psi=1}^{19}\{\mathrm{a}-(2 \Psi-1)\}$

$4688307 a^{2} b^{17}-2686866 a^{3} b^{17}-60591 b^{18}+79781 a b^{18}-73112 a^{2} b^{18}+361 b^{19}-702 a b^{19}-b^{20}$

$\prod_{\Psi_{1}}^{19}\{a-(2 \Psi-1)\}$

$\prod_{\Psi=1}^{19}\{\mathrm{a}-(2 \Psi-1)\}$

$+\frac{\Gamma\left(\frac{b+1}{2}\right)}{\Gamma\left(\frac{a-36}{2}\right)}\left\{\frac{13097439371123680592250 a-22153940827063831976400 a^{2}+17925525907364074526550 a^{3}}{\prod^{10}=1\{a-2 \Omega\}}\right.$

$-7567822489717658616192 a^{4}+2458271220624423404328 a^{5}-479271213946622028480 a^{6}$

$\prod_{\Omega=1}^{18}\{a-2 \Omega\}$

$84654065201507029112 a^{7}-9092335240513010304 a^{8}+982146241369204236 a^{9}-62317491573823200 a^{10}$

$\prod_{\Omega=1}^{18}\{a-2 \Omega\}$

$4336214993731764 a^{11}-165231363762816 a^{12}+7512309428744 a^{13}-166874823360 a^{14}+4857736536 a^{15}$ $\prod_{\Omega=1}^{18}\{\mathrm{a}-2 \Omega\}$

$4336214993731764 a^{11}-165231363762816 a^{12}+7512309428744 a^{13}-166874823360 a^{14}+4857736536 a^{15}$ $\prod_{\Omega=1}^{18}\{a-2 \Omega\}$

$-56930688 a^{16}+986442 a^{17}-4560 a^{18}+38 a^{19}-13097439371123680592250 b+23139738814018920063390 a^{2} b$ $\prod_{\Omega=1}^{18}\{a-2 \Omega\}$

$-21516156363100184047872 a^{3} b+11827389742977955137336 a^{4} b-3325546317323479116032 a^{5} b$ $\prod_{\Omega=1}^{18}\{a-2 \Omega\}$

$832659328882303559224 a^{6} b-114754994980386645248 a^{7} b+16598022165655290516 a^{8} b$

$\prod_{\Omega=1}^{18}\{a-2 \Omega\}$

$-1276900600798194944 \mathrm{a}^{9} \mathrm{~b}+116930668507644420 \mathrm{a}^{10} \mathrm{~b}-5224908392674048 \mathrm{a}^{11} \mathrm{~b}$

$\prod_{\Omega=1}^{18}\{a-2 \Omega\}$

$314648876615320 a^{12} b-8027640199936 a^{13} b+319151847768 a^{14} b-4249253632 a^{15} b+108006678 a^{16} b$ $\prod_{\Omega=1}^{18}\{a-2 \Omega\}$

$-569088 a^{17} b+8398 a^{18} b+22153940827063831976400 b^{2}-23139738814018920063390 a b 2$

$\prod_{\Omega=1}^{18}\{a-2 \Omega\}$

$9933711099522196655760 \mathrm{a}^{3} \mathrm{~b}^{2}-5738820296938637651392 \mathrm{a}^{4} \mathrm{~b}^{2}+2322425070729799904856 \mathrm{a}^{5} \mathrm{~b}^{2}$

$\prod^{18}-\{a-2 \Omega\}$

$-448268772128253649664 a^{6} b^{2}+89755345390166601392 a^{7} b^{2}-8730337031956713888 a^{8} b^{2}$ $\prod_{\Omega=1}^{18}\{a-2 \Omega\}$

$1055369472607482604 \mathrm{a}^{9} \mathrm{~b}^{2}-56752913346162176 \mathrm{a}^{10} \mathrm{~b}^{2}+4458667436531568 \mathrm{a}^{11} \mathrm{~b}^{2}-133022238102208 \mathrm{a}^{12} \mathrm{~b}^{2}$ $\prod_{\Omega=1}^{18}\{\mathrm{a}-2 \Omega\}$

$6976042787224 a^{13} b^{2}-106790311680 a^{14} b^{2}+3716282960 a^{15} b^{2}-22372272 a^{16} b^{2}+493506 a^{17} b^{2}$ $\prod_{\Omega=1}^{18}\{a-2 \Omega\}$

$+17925525907364074526550 b^{3}+21516156363100184047872 a^{3}-9933711099522196655760 a^{2} b^{3}$ $\prod_{\Omega=1}^{18}\{\mathrm{a}-2 \Omega\}$

$1600403274473963897496 a^{4} b^{3}-607219120610904465664 a^{5} b^{3}+190415570648151178512 a^{6} b^{3}$ $\prod_{\Omega=1}^{18}\{a-2 \Omega\}$

$-25391094996880797184 a^{7} b^{3}+4176061103280434940 a^{8} b^{3}-280686885125320960 a^{9} b^{3}$ $\prod_{\Omega=1}^{18}\{a-2 \Omega\}$

$28815447847634448 a^{10} b^{3}-1028399437485056 a^{11} b^{3}+69991902775704 a^{12} b^{3}-1250170208000 a^{13} b^{3}$ $\prod_{\Omega=1}^{18}\{a-2 \Omega\}$

$57384877680 a^{14} b^{3}-397729280 a^{15} b^{3}+12118314 a^{16} b^{3}+7567822489717658616192 b^{4}$ $\prod_{\Omega=1}^{18}\{a-2 \Omega\}$

$+-11827389742977955137336 a^{4}+5738820296938637651392 a^{2} b^{4}-1600403274473963897496 a^{3} b^{4}$ $\prod_{\Omega=1}^{18}\{a-2 \Omega\}$

$116886064094067881704 a^{5} b^{4}-29718620638310828224 a^{6} b^{4}+7483938508477753608 a^{7} b^{4}$ $\prod_{\Omega=1}^{18}\{a-2 \Omega\}$

$-678604057567962240 a^{8} b^{4}+93576506319484760 a^{9} b^{4}-4136807371336640 a^{10} b^{4}+365316837457208 a^{11} b^{4}$ $\prod_{\Omega=1}^{18}\{\mathrm{a}-2 \Omega\}$

$-7769044890880 a^{12} b^{4}+461716368120 a^{13} b^{4}-3728712000 a^{14} b^{4}+150391384 a^{15} b^{4}$

$\prod_{\Omega=1}^{18}\{a-2 \Omega\}$ 
Vol. 1, Issue 1, pp. 022-027

$+-2458271220624423404328 b^{5}+3325546317323479116032 a b^{5}-2322425070729799904856 a^{2} b^{5}$ $\prod_{\Omega=1}^{18}\{a-2 \Omega\}$

$607219120610904465664 \mathrm{a}^{3} \mathrm{~b}-116886064094067881704 \mathrm{a}^{4} \mathrm{~b}^{5}+4258621100102048680 \mathrm{a}^{6} \mathrm{~b}^{5}$ $\prod_{\Omega=1}^{18}\{a-2 \Omega\}$

$+-720221439853014528 a^{7} b^{5}+149549567547044808 a^{8} b^{5}-8809810923513600 a^{9} b^{5}+1035181358595384 a^{10} b^{5}$ $\prod_{\Omega=1}^{18}\{a-2 \Omega\}$

$+-27078647022336 a^{11} b^{5}+2078656264776 a^{12} b^{5}-19917058560 a^{13} b^{5}+1040310648 a^{14} b^{5}$

$\prod_{\Omega=1}^{18}\{a-2 \Omega\}$

$479271213946622028480 b^{6}-832659328882303559224 a^{6}+448268772128253649664 a^{2} b^{6}$ $\prod_{\Omega=1}^{18}\{\mathrm{a}-2 \Omega\}$

$-190415570648151178512 a^{3} b^{6}+29718620638310828224 a^{4} b^{6}-4258621100102048680 a^{5} b^{6}$ $\prod_{\Omega=1}^{18}\{a-2 \Omega\}$

$80696899873601440 a^{7} b^{6}-8721445194990528 a^{8} b^{6}+1523988869787384 a^{9} b^{6}-52617684863744 a^{10} b^{6}$ $\prod_{\Omega=1}^{18}\{a-2 \Omega\}$

$5338008453232 a^{11} b^{6}-62572758976 a^{12} b^{6}+4211628008 a^{13} b^{6}-84654065201507029112 b^{7}$

$\prod_{\Omega=1}^{18}\{a-2 \Omega\}$

$114754994980386645248 \mathrm{ab}^{7}-89755345390166601392 \mathrm{a}^{2} \mathrm{~b}^{7}+25391094996880797184 \mathrm{a}^{3} \mathrm{~b}^{7}$

$\prod_{\Omega=1}^{18}\{a-2 \Omega\}$

$+7483938508477753608 a^{4} b^{7}+720221439853014528 a^{5} b^{7}-80696899873601440 a^{6} b^{7}+791050371234552 a^{8} b^{7}$ $\prod_{\Omega=1}^{18}\{a-2 \Omega\}$

$-49480910519040 a^{9} b^{7}+7395423461712 a^{10} b^{7}-113577444864 a^{11} b^{7}+10056336264 a^{12} b^{7}$ $\prod_{\Omega=1}^{18}\{a-2 \Omega\}$

$9092335240513010304 b^{8}-16598022165655290516 a b^{8}+8730337031956713888 a^{2} b^{8}$

$\prod_{\Omega=1}^{18}\{a-2 \Omega\}$

$+4176061103280434940 a^{3} b^{8}+678604057567962240 a^{4} b^{8}-149549567547044808 a^{5} b^{8}$

$\prod_{\Omega=1}^{18}\{a-2 \Omega\}$

$8721445194990528 a^{6} b^{8}-791050371234552 a^{7} b^{8}+3728705795100 a^{9} b^{8}-102742754400 a^{10} b^{8}$

$\prod_{\Omega=1}^{18}\{a-2 \Omega\}$

$+13309856820 a^{11} b^{8}-982146241369204236 b^{9}+1276900600798194944 a b^{9}-1055369472607482604 a^{2} b^{9}$ $\prod_{\Omega=1}^{18}\{\mathrm{a}-2 \Omega\}$

$280686885125320960 a^{3} b^{9}-93576506319484760 a^{4} b^{9}+8809810923513600 a^{5} b^{9}-1523988869787384 a^{6} b^{9}$

$\prod_{\Omega=1}^{18}\{\mathrm{a}-2 \Omega\}$

$49480910519040 a^{7} b^{9}-3728705795100 a^{8} b^{9}+6564120420 a^{10} b^{9}+62317491573823200 b^{10}$

$\prod_{\Omega=1}^{18}\{a-2 \Omega\}$

$-116930668507644420 a b^{10}+56752913346162176 a^{2} b^{10}-28815447847634448 a^{3} b^{10}$

$\prod_{\Omega=1}^{18}\{a-2 \Omega\}$

$4136807371336640 a^{4} b^{10}-1035181358595384 a^{5} b^{10}+52617684863744 a^{6} b^{10}-7395423461712 a^{7} b^{10}$

$\prod_{\Omega=1}^{18}\{\mathrm{a}-2 \Omega\}$

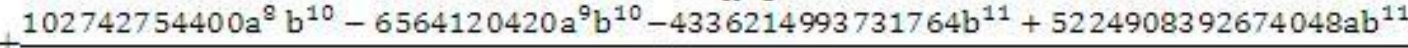

$\prod_{\Omega=1}^{18}\{a-2 \Omega\}$

$-4458667436531568 a^{2} b^{11}+1028399437485056 a^{3} b^{11}-365316837457208 a^{4} b^{11}+27078647022336 a^{5} b^{11}$

$\prod_{\Omega=1}^{18}\{a-2 \Omega\}$

$-5338008453232 a^{6} b^{11}+113577444864 a^{7} b^{11}-13309856820 a^{8} b^{11}+165231363762816 b^{12}$

$$
\prod_{\Omega=1}^{18}\{a-2 \Omega\}
$$

$-314648876615320 a b^{12}+133022238102208 a^{2} b^{12}-69991902775704 a^{3} b^{12}+7769044890880 a^{4} b^{12}$

$\prod_{\Omega=1}^{18}\{a-2 \Omega\}$

$-2078656264776 a^{5} b^{12}+62572758976 a^{6} b^{12}-10056336264 a^{7} b^{12}-7512309428744 b^{13}$

$\prod_{\Omega=1}^{18}\{\mathrm{a}-2 \Omega\}$

$8027640199936 a b^{13}-6976042787224 a^{2} b^{13}+1250170208000 a^{3} b^{13}-461716368120 a^{4} b^{13}+19917058560 a^{5} b^{13}$ $\prod_{\Omega=1}^{18}\{\mathrm{a}-2 \Omega\}$

$-4211628008 a^{6} b^{13}+166874823360 b^{14}-319151847768 a b^{14}+106790311680 a^{2} b^{14}-57384877680 a^{3} b^{14}$ $\prod_{\Omega=1}^{18}\{\mathrm{a}-2 \Omega\}$

$3728712000 a^{4} b^{14}-1040310648 a^{5} b^{14}-4857736536 b^{15}+4249253632 a b^{15}-3716282960 a^{2} b^{15}$ $\prod_{\Omega=1}^{18}\{a-2 \Omega\}$ 
SALAHUDDIN / IOSR Journal of Engineering (IOSRJEN)

www.iosrjen.org

Vol. 1, Issue 1, pp. 022-027

$+397729280 a^{3} b^{15}-150391384 a^{4} b^{15}+56930688 b^{16}-108006678 a b^{16}+22372272 a^{2} b^{16}-12118314 a^{3} b^{16}$

$\prod_{\Omega=1}^{18}\{a-2 \Omega\}$

$\left.\left.+\frac{-986442 b^{17}+569088 a b^{17}-493506 a^{2} b^{17}+4560 b^{18}-8398 a b^{18}-38 b^{19}}{\prod_{\Omega=1}^{18}\{a-2 \Omega\}}\right\}\right]$

\section{DERIVATION OF THE MAIN SUMMATION FORMULA:}

Putting $c=\frac{a+b-37}{2}$ and $z=\frac{1}{2}$ in equation (2), we get

$(\mathrm{a}-\mathrm{b}){ }_{2} \mathrm{~F}_{1}\left(\mathrm{a}, \mathrm{b} ; \frac{\mathrm{a}+\mathrm{b}-37}{2} ; \frac{\mathbf{1}}{2}\right)=(\mathrm{a}-\mathrm{b}-37){ }_{2} \mathrm{~F}_{1}\left(\mathrm{a}, \mathrm{b}-1 ; \frac{\mathrm{a}+\mathrm{b}-37}{2} ; \frac{\mathbf{1}}{2}\right)+(\mathrm{a}-\mathrm{b}+37){ }_{2} \mathrm{~F}_{1}\left(\mathrm{a}-1, \mathrm{~b} ; \frac{\mathrm{a}+\mathrm{b}-37}{2} ; \frac{\mathbf{1}}{2}\right)$

Now using the same parallel method of Ref [4], the summation formula is obtained.

\section{REFERENCES}

[1]. Abramowitz, Milton., A and Stegun, Irene ; Handbook of Mathematical Functions with Formulas, Graphs, and Mathematical Tables. Na-

tional Bureau of Standards, 1970.

[2] . Arora, Asish, Singh, Rahul, Salahuddin ; Development of a family of summation formulae of half argument using Gauss and Bailey theorems, Journal of Rajasthan academy of Physical Sciences. ,7(2008), 335-342

[3]. Bells, Richard., Wong, Roderick ; Special Functions , A Graduate Text. Cambridge Studies in Advanced Mathematics, 2010.

[4]. Salahuddin. ; Three Summation Formulae using Contiguous Relation and Related to Hypergeometric function , International Journal of Mathematical Archieve., 2(2011), 518-520. 